\title{
Endoluminal treatment for venous vascular complications of malignant tumors
}

\author{
LIANG XIAO, JIA-JIE TONG and JING SHEN
}

Department of Radiology, First Hospital of China Medical University, Shenyang, Liaoning 110001, P.R. China

Received March 16, 2012; Accepted May 23, 2012

DOI: $10.3892 / \mathrm{etm} .2012 .589$

\begin{abstract}
The aim of this study was to explore the efficacy and safety of interventional treatment for venous vascular complications of malignant tumors. Sixty-one patients with venous vascular complications of malignant tumors were treated from May 2002 to May 2009; 37 men and 24 women with mean age 57.8 years (33-82 years). Lesions included acute deep vein thrombosis $(n=18)$; venous stenosis or occlusion $(n=32)$; tumor embolus in vein $(n=11)$. The interventional therapeutic operations included vena cava filter implantation, transcatheter thrombolytic therapy, recanalization, percutaneous transluminal angioplasty (PTA) and stenting. The success rate of thrombolysis and stent implantation, the clinical success rate, complications, recurrence rate of the treated region and survival duration were recorded. Eighteen patients accepted filter and thrombolytic therapy with a success rate of $100 \%$; total urokinase dosage was $7.42 \pm 1.49(4.5-10)$ million units. Symptoms disappeared $(n=15)$, were palliated $(n=3)$ and thrombi were completely dissolved $(n=2)$, almost completely dissolved $(n=8,>90 \%)$, partially dissolved $(n=6,50-90 \%)$ and not dissolved $(\mathrm{n}=2,<50 \%)$. No pulmonary embolism emerged after the operation. Forty-three patients accepted recanalization, PTA and stent therapy with a success rate of $95.3 \%(41 / 43)$. Symptoms disappeared $(n=25)$, were palliated $(n=16)$ and did not change $(n=2) 3$ days following the operation. There were no severe complications during the procedure. During followup, 12 patients again suffered symptoms of venous occlusion and 47 patients died of tumor aggravation without symptom recurrence. As a result, interventional therapy has advantages including smaller injuries, well tolerance, high success rate, quick palliation of symptoms and superior clinical efficacy in the treatment of venous vascular complications for malignant tumors.
\end{abstract}

Correspondence to: Professor Liang Xiao, Department of Radiology, First Hospital of China Medical University, 155 Nanjing North Road, Shenyang 110001, Liaoning, P.R. China

E-mail: xiaoliangcmu@yahoo.com.cn

Key words: malignant tumor, venous occlusion, transcatheter thrombolysis, stents

\section{Introduction}

Growth of malignant lesions surrounding the vena cava or large veins often results in stenosis or obstruction of the venous vascular lumen and symptoms of venous congestion. Some malignant tumors may invade the vascular wall to form a tumor embolus or induce thrombosis in the adjacent vein. Based on the location and extent of the venous obstruction, this may result in pain and severe edema of the face, neck, torso and limbs. Other severe symptoms such as dyspnea, dysphagia and ascites may also occur. The severity of symptoms is increased by liver dysfunction and gastrointestinal bleeding from portal tumor embolus, renal dysfunction from inferior vena cava (IVC) occlusion, airway obstruction from laryngeal or bronchial edema and coma from cerebral edema.

Traditional conservative therapy with anticoagulation may help to slow the progression of symptom aggravations, but may not be efficient for rapid symptom relief, and severe symptoms frequently persist. In addition, surgical treatment of these symptoms in patients with malignant disease generally has poor results, and therefore more microinvasive therapies are advocated. Endovascular intervention provides an effective method for venous vascular complications of malignant tumors, with good tolerance and few injuries. Catheter-directed thrombolytic therapy has become widely accepted as a valuable treatment option in patients with acute deep vein thrombosis (DVT) (1-5). Self-expandable metallic stent placement in the vena cava and large veins at the site of such lesions is widely accepted as a means to improve the quality of life of patients with advanced malignant disease (6-8).

The purpose of this study was to evaluate the efficacy and safety of our experience with vena cava filter implantation, catheter-directed thrombolytic therapy, recanalization, percutaneous transluminal angioplasty (PTA) and stent placement in patients with venous vascular complications of malignant tumors.

\section{Materials and methods}

Patients. Between May 2002 and May 2009, 61 consecutive patients with venous vascular complications of malignant tumors ( 37 male, 24 female; mean age, 57.8 years; age range, 33-82 years) from the First Hospital of China Medical University (Shenyang, China) were enrolled in this study. After providing a complete description of the study to the patients, 


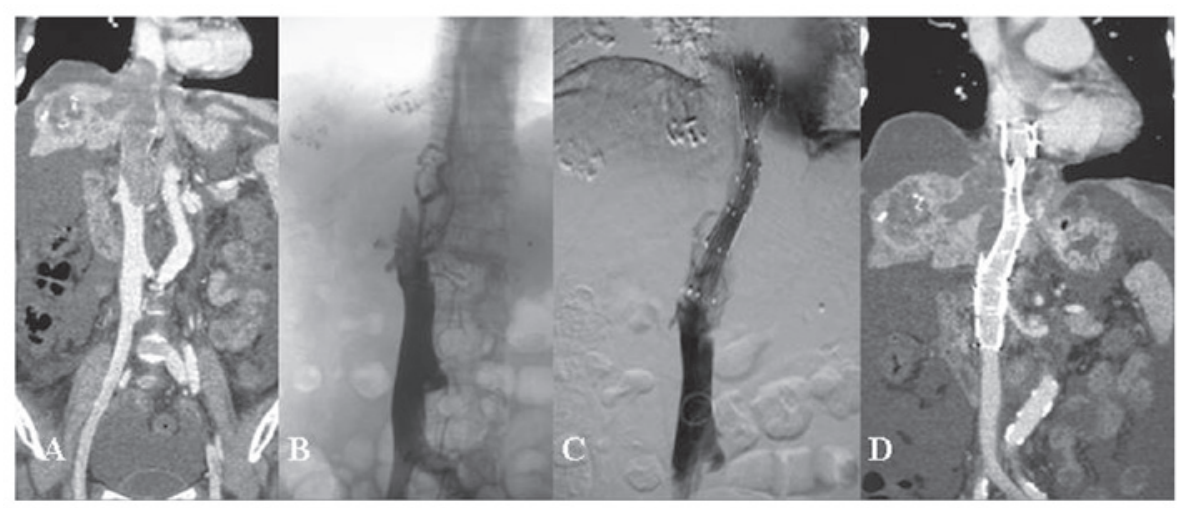

Figure 1. A 67-year-old female primary hepatic carcinoma patient with inferior vena cava occlusion. (A) CT-angiography and (B) cavogram of the inferior vena cava demonstrated tumor embolus in the hepatic vein and inferior vena cava occlusion. After recanalization of the inferior vena cava and $3 \mathrm{Z}$-stents were implanted, (C) cavogram and (D) inferior vena cava CT-angiography demonstrated that the contrast agent in the IVC entered fluently in the right atrium through the stents. This patient died of hepatorenal syndrome 6 months later but without recurrence of the IVC obstruction.

written informed consent was obtained in accordance with the National Health and Medical Research Council guidelines. The study was approved by the Ethics Committee of our hospital.

All patients experienced venous obstruction by malignant tumors and the symptoms included pain and swelling of the torso and limbs $(n=38)$, pain and swelling of the face and neck $(n=25)$, varicosis of the torso $(n=22)$, headache $(n=8)$, ascites $(n=6)$, dyspnea $(n=5)$, dysphagia $(n=2)$, liver dysfunction $(n=2)$, gastrointestinal bleeding $(n=2)$ and renal dysfunction $(n=1)$. Vascular ultrasound or computed tomography (CT) scans were performed before interventional therapy and revealed unresectable malignant venous obstruction in all patients. Venous stenosis or occlusion was detected in 32 cases, acute deep vein thrombosis in 18 cases and tumor embolus in the vein in 11 cases. Involved veins included the superior vena cava (SVC) (29 cases), upper extremity veins (9 cases), iliac veins ( 9 cases), IVC ( 7 cases), portal veins (4 cases) and jugular veins (3 cases). Underlying obstructing tumors included lung cancer and lymph node metastasis $(\mathrm{n}=25)$, primary hepatic carcinoma $(n=11)$, colorectal cancer $(n=9)$, lymph node metastasis $(n=7)$, malignant thymoma $(n=6)$ and thyroid carcinoma $(n=3)$. All malignancies were diagnosed by pathological and/or imaging findings in addition to clinical features.

\section{Procedure}

Filter implantation and catheter-directed thrombolytic therapy. Utilizing a femoral vein approach, a 5-Fr pig-tail catheter was placed into the SVC or IVC. A cavogram was performed to ensure that the vena cava was free of thrombus and to document the diameter of the vena cava. An OptEase filter (Cordis Corporation, Miami Lakes, FL, USA) or a Günther Tulip filter (Vena Cava MReye filter set; William Cook Europe, Bjaeverskov, Denmark) was implanted into the SVC or IVC.

The presence of contraindications to percutaneous thrombolysis, including metastatic central nervous system malignancy, coagulopathy, occurrence of stroke $<3$ months prior, surgery $<1$ month prior, or gastrointestinal bleeding was assessed and patients were excluded. Patients at risk for cerebrovascular accidents or cerebral metastasis were further evaluated by CT before thrombolysis treatments. After excluding pulmonary embolism by pulmonary arteriogram, a 5-F curved catheter over a 0.035 -inch hydrophilic guide wire was inserted near the thrombosed region. The guide wire was pushed firstly towards and into the thrombus with the support from the catheter. Then, the catheter entered the thrombus over the guide wire. A veinogram was performed to ensure the extent of the thrombus and collateral veins. Subsequently, a thrombolytic catheter (Unifuse, Angiodynamics, Inc., Queensbury, NY, USA) with a 20-cm- or 30-cm long sidehole was replaced into the thrombus. Urokinase (500,000 IU) was infused for $2 \mathrm{~h}$ twice every day through the thrombolytic catheter. Systemic anticoagulant therapy was administered by intravenously injecting $50 \mathrm{IU} / \mathrm{kg}$ heparin every $6 \mathrm{~h}$. Repeated veinogram was performed every 3-4 days during thrombolysis therapy. According to the extent of the residual thrombus, the location of the thrombolytic catheter was adjusted.

After thrombolysis therapy, oral warfarin was administered to maintain an international normalized ratio of 2-2.5 during follow-up.

Recanalization, PTA and stent. In order to demonstrate the extent of the obstructed vein, a 5-Fr catheter was percutaneously delivered from the femoral vein or fluent portal vein branch and introduced into the distal region of the obstructed vein after a 0.035 -inch hydrophilic guide wire was placed beyond the obstructing lesion. In the event that it was impossible to cross the SVC with a guide wire via the femoral route, the lesion was crossed with a hydrophilic guide wire via the brachial route. After injection of heparin (50 IU/kg), balloon angioplasty was performed (balloon, $8 \times 40 \mathrm{~mm}$ ) to allow easy transition of the introducer sheath carrying the stent.

The diameter of the Z-stent implanted in the vena cava was 20-24 $\mathrm{mm}$ and the length of the single Z-stent was $70 \mathrm{~mm}$ (Fig. 1). The diameter of the Luminexx self-expandable stent implanted in the iliac vein was $10-12 \mathrm{~mm}$ and the length of the stent was 40-80 mm (Fig. 2). The diameter of the Fluency stentgraft implanted in the portal vein was $10 \mathrm{~mm}$ and the length of the stent was $60-80 \mathrm{~mm}$ (Fig. 3). The stent was customized for each patient, taking into consideration the length and diameter of the obstructing lesions and the diameter of the normal regions of the vein near the obstructing lesion. The stent was implanted accordingly so that it extended over both sides of 


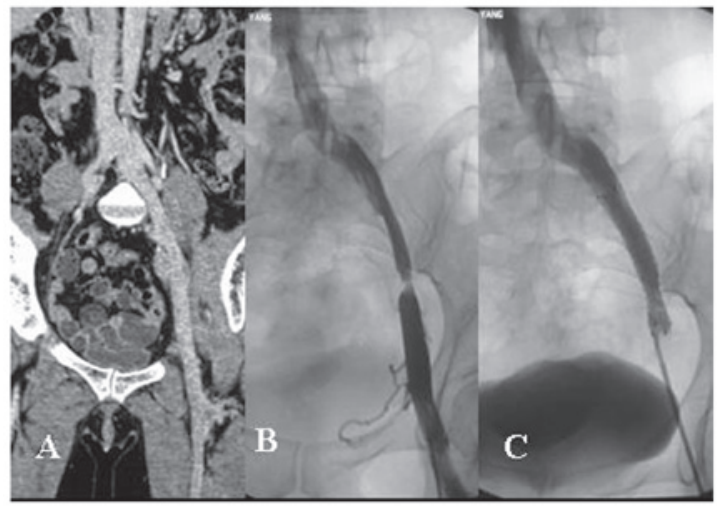

Figure 2. A 52-year-old female rectal cancer patient with a left external iliac vein stenosis. (A) The lower extremity deep vein CT-angiography demonstrated a lesion around the left external iliac vein. (B) The veinogram of the left iliofemoral vein demonstrated a centripetal stenosis of the left external iliac vein and obstructed flow in the left iliofemoral vein. (C) After a 10-mmdiameter stent was implanted, repeated veinogram demonstrated fluent flow in the left iliofemoral vein. This patient died of liver metastasis and liver failure 21 months later but without recurrence of the venous obstruction.

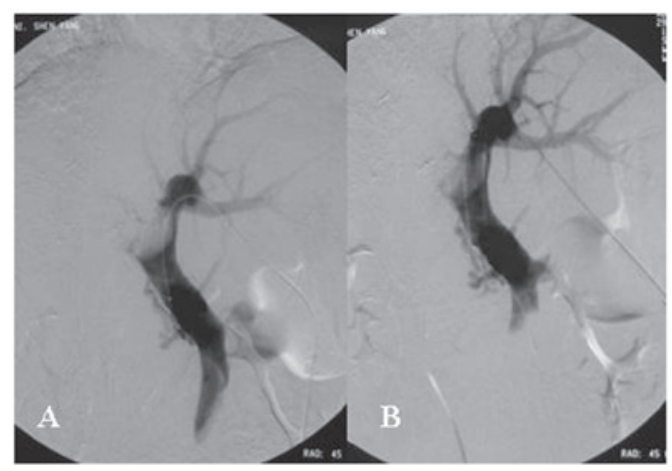

Figure 3. A 47-year-old male primary hepatic carcinoma patient with large tumor embolus in the portal vein. (A) The portogram on right anterior oblique $45^{\circ}$ demonstrated tumor embolus in the portal vein, complete occlusion of the right portal branch and stenosis of the main and left portal vein. (B) After a 10-mm-diameter stent-graft was implanted, repeated portogram demonstrated that the left portal branches received more blood perfusion than that prior to operation. This patient died of liver failure 12 months later but without recurrence of portal venous obstruction.

the obstructing lesion by $1-2 \mathrm{~cm}$. For longer lesions, two or three stents were implanted. If stent dilatation was insufficient, post-dilatation was performed with balloon.

After the procedure, systemic anticoagulant therapy was administered by intravenously injecting $50 \mathrm{IU} / \mathrm{kg}$ heparin every 6 h for 5 days. Subsequently, oral warfarin was administered to maintain an international normalized ratio of 2-2.5 at least for 3 months.

Study endpoints and definitions. The study endpoints were the success rate of thrombolysis and stent implantation, the total urokinase dosage, the clinical success rate, the operationrelated complication rate, the recurrence rate of the treated region and the survival duration without recurrence of symptoms.

Thrombolysis was defined as complete (grade III) (Fig. 4), nearly complete (>90\%, grade IIa), partial (50-90\%, grade IIb)

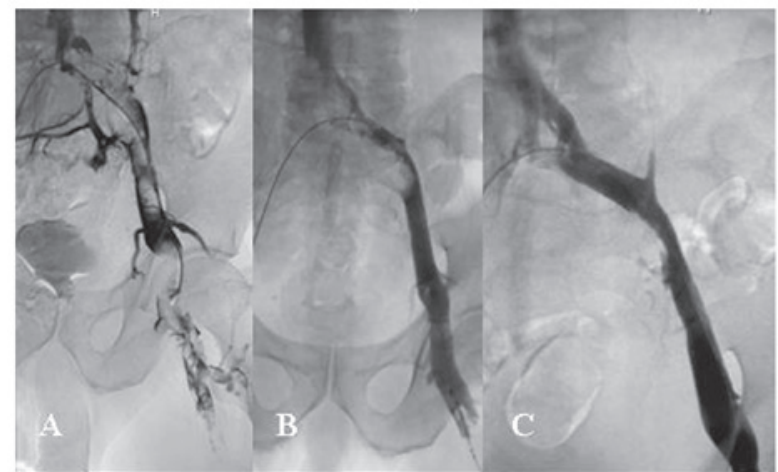

Figure 4. A 62-year-old male sigmoid colon carcinoma patient with left lower extremity deep vein thrombosis. (A) The left iliac veinogram demonstrated full thrombus in the left iliofemoral vein. (B) Repeated veinogram after 7-day transcatheter thrombolysis demonstrated that thrombus in the external iliac vein was completely dissolved. (C) Repeated veinogram after 10-day transcatheter thrombolysis demonstrated that thrombus in the common iliac vein was completely dissolved and that the lateral wall of the external iliac vein was invaded by the tumor. This patient died of enterobrosis 25 months later but without recurrence of the venous obstruction.

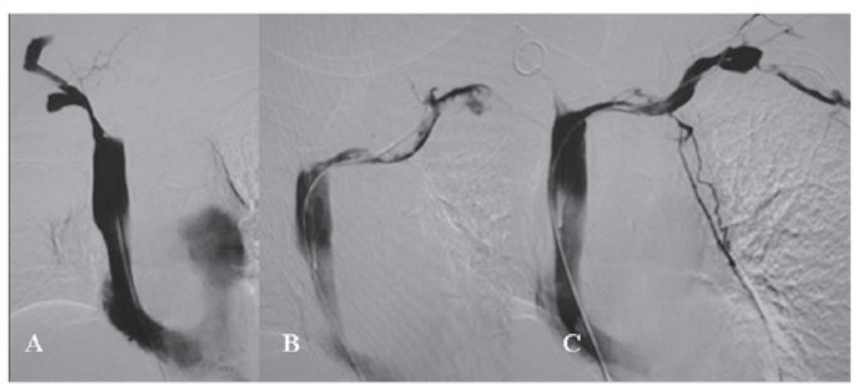

Figure 5. A 45-year-old female thyroid carcinoma patient with right innominate vein stenosis and left upper extremity deep vein thrombosis. (A) The upper cavogram demonstrated superior vena cava fluent, right innominate vein stenosis and left innominate vein occlusion. (B) After recanalization of the left upper extremity deep vein, veinogram demonstrated full thrombus in the left upper extremity vein. (C) Repeated veinogram after 7-day transcatheter thrombolysis demonstrated that thrombus in the left innominate vein was partially dissolved and that the thrombus in the left subclavian vein was not dissolved. This patient died of suffocation 15 months later but without recurrence of the venous obstruction.

or no $(<50 \%$, grade I) (Fig. 5) thrombolytic effect at the final veinogram (1). Technical success of the stent implantation was defined as successful placement of the stent into the obstructing lesion. Clinical success was defined as the complete alleviation of symptoms.

Follow-up. Patency of the treated region was assessed by performing Doppler ultrasound every 3 months after operation or at any time if the symptoms of venous obstruction recurred. The clinical courses after operation and the causes of death were studied from clinical records until October 1, 2011.

\section{Results}

Initial clinical results. Technical success was achieved in all 18 patients who accepted filter and thrombolytic therapy (100\%). The total urokinase dosage was $7.42 \pm 1.49(4.5-10)$ million units. 
Thrombi in 2 patients were completely dissolved (grade III), in 8 patients nearly completely dissolved (grade IIa), in 6 patients partially dissolved (grade IIb) and in 2 patients not dissolved (grade I) (Fig. 5). No pulmonary embolism emerged after operations. The clinical success rate was $83.3 \%(15 / 18)$ when thrombolytic therapy was terminated. The symptoms of 3 patients were palliated.

Among the 43 patients who accepted recanalization, PTA and stent therapy, the technical success rate was $95.3 \%$ (41/43). SVC obstructions in 2 patients were not successfully recanalized through femoral and brachial approaches. The symptoms of venous obstruction were persistent. One patient died due to respiratory failure secondary to hemoptysis and one due to suffocation secondary to the invasion of the lung cancer into the bronchus 2 weeks and 1 month after operation. The clinical success rate was $61.0 \%$ (25/41) within 3 days after recanalization, PTA and stent therapy. The symptoms of 16 patients were palliated.

Follow-up results. All 61 patients died, and the longest follow-up period was 32 months. Among the 18 patients who received filter and thrombolytic therapy, the survival rate of 14 of these patients in whom there was no recurrence of venous obstruction until death ranged from 6 to 32 months (median, 16.5 months). The survival rate of 4 patients in whom recurrence occurred ranged from 5 to 20 months (median, 9.5 months). Three patients recurred after stopping warfarin and 1 patient recurred during oral warfarin. The causes of death included lung metastasis $(n=5)$, liver metastasis $(n=5)$, cerebral metastasis $(n=3)$, cachexia $(n=2)$, suffocation $(n=2)$ and enterobrosis $(\mathrm{n}=1)$.

Among the 41 patients who successfully accepted recanalization, PTA and stent therapy, the survival rate of 33 patients in whom there was no recurrence of venous obstruction until death ranged from 3 to 21 months $(12.9 \pm 4.6$; median, 13.0 months). The survival rate of 8 patients in whom recurrence occurred ranged from 2 to 18 months $(10.1 \pm 4.5$, median, 10.0 months). The causes of death included respiratory failure $(n=12)$, suffocation $(n=8)$, cerebral metastasis $(n=6)$, liver failure $(n=5)$, lung metastasis $(n=4)$, hepatic encephalopathy $(n=2)$, hepatorenal syndrome $(n=2)$, enterobrosis $(n=2)$ and gastrointestinal bleeding $(n=1)$.

Complications. There were no severe complications during the procedure. Six patients $(9.8 \%)$ experienced complications, including 5 patients with temporary local pain and 1 patient with hematuria who revived after stopping thrombolytic therapy.

\section{Discussion}

Although vascular complications of malignancies and concomitant hemodynamic changes are rarely direct causes of death, they are usually facilitative factors of tumor deterioration and predictors of poor prognosis. The aim of treatments for malignant venous complications is to relieve venous obstructive symptoms, improve the quality of life of patients and increase the survival rate. Present therapies for this condition include radiotherapy, chemotherapy, surgical therapy, endovascular therapy and anticoagulant therapy. Radiotherapy and chemotherapy may induce the reduction in tumor bulk and reduce the extent of venous invasion and compression through killing and injuring tumor cells. These therapies may radically cure malignant tumors and their venous complications, but symptoms of venous obstructive are usually noted at the early stage due to tumor-cell swelling. Endovascular therapy may reconstruct fluent lumen, remove the thrombus and quickly relieve the venous congestion. The ideal therapeutic regimen for malignant venous complications is endovascular therapy followed by radiotherapy, chemotherapy and anticoagulant therapy $(9,10)$.

Our study demonstrates that catheter-directed thrombolytic therapy for thrombosis of large veins is quite effective with thrombus dissolution of grade II or III in $88.9 \%$ of cases. This result is in accordance with the results obtained by Kee et al (2) for SVC syndrome, Kim et al (11) for upper and lower limb DVT and Maleux et al (12) for thoracic deep vein thrombosis.

Due to concerns of a risk of bleeding, the presence of malignancy has been considered an exclusion criterion in several clinical trials dealing with catheter-directed thrombolytic therapy $(1,13)$. However, Kim et al (11) and Maleux et al (12) found no significant difference between cancer and noncancer patients in the safety of thrombolytic therapy for DVT in the lower and upper limbs and in the thoracic cavity.

Maleux et al (12) reported that an intracranial hemorrhage occurred in a cancer patient with an unknown and asymptomatic cerebral metastasis during catheter-directed thrombolytic therapy for thoracic deep vein thrombosis in a retrospective trial. This fatal complication underlines the need for brain imaging before starting thrombolytic therapy, especially for cancer patients. No intracranial bleeding occurred in our study as patients with cerebral metastasis and cerebrovascular accidents were excluded from thrombolytic therapy.

Patients with malignancy often encounter a hypercoagulable state, so long-term anticoagulant therapy is essential after successful catheter-directed thrombolytic therapy. In our study, all 3 patients who ceased anticoagulant therapy encountered re-thrombosis. Only 1 of 15 patients who continued anticoagulant therapy encountered re-thrombosis. No bleeding occurred during follow-up and anticoagulant therapy. Hutten et al (14) and Prandoni et al (15) found a 2- to 6-fold increased risk of bleeding in cancer patients compared with non-cancer patients during systemic anticoagulation.

Venous obstructions significantly impact the quality of life patients, and palliative therapies may be indicated even for patients with malignancies with relatively short life expectancies. Radiation and chemotherapy are only modestly effective for vein obstruction secondary to malignant tumors, thus stent placement represents a less invasive but effective therapy for relieving vein obstruction and increasing the quality of life of these patients $(2,8,10,16-23)$. Due to risk factors, SVC occlusion is usually more serious than that of IVC and iliac veins for similar venous obstructive symptoms. The difficulty in the recanalization of the SVC is greater than that in other veins. To increase the success rate of recanalization, the brachial route should be used when the lesion is crossed with a hydrophilic guide wire via the femoral route. In the present study, all 2 unsuccessful recanalizations occurred in SVC occlusion patients. 
If dilatation of the implanted stent in the superior vena cava is insufficient, post-dilatation should be moderately performed with balloon. Excess dilatation may induce adjacent lumen compression or collapse for example presenting as dyspnea due to tracheal collapse. There are various complications (24-27) related to vena cava stent implantation, including pulmonary edema resulting from a high venous return, stent migration, pulmonary embolus, cardiac tamponade and local pain. To avoid stent migration, the diameter of the stent should be greater than that of the normal vein, and the length of the stent should be 2-4 cm longer than that of the lesion.

The objective of stent-graft implantation for intrahepatic portal vein tumor embolus is to assure adequate blood supply to the non-tumor side of the liver lobe, to support normal hepatic function and to avoid tumor embolus to intrude the contralateral portal branch. In our study, the portal right or left branch was occluded completely by a tumor embolus and the embolus intruded the contralateral portal branch and/or portal major branch in all 4 patients who received portal vein stentgraft implantation. Stent-grafts were implanted between the portal major branch and the contralateral portal branch. Due to the 10-mm diameter, the distal segment of the stent-graft could not be attached tightly to the vascular wall. The anchoring area of the $10 \mathrm{~mm}$ stent-graft was located at the contralateral portal branch. In order to prevent tumor embolus overgrowth, the stent-graft was placed over both sides of the obstructing lesion by $2 \mathrm{~cm}$. The diameter of the uncovered stents for malignant extrinsic portal vein stenosis or occlusion were determined according to the diameter of the involved portal vein with caution to oversize the stent diameter by $1-2 \mathrm{~mm}$ (23).

To assure enough space for anchoring area of the stent, the puncture entrance of the portal branch should be $>2 \mathrm{~cm}$ from the tumor embolus. To show the relationship between the left portal branch and the main trunk, the portogram should be performed on right anterior oblique $30-45^{\circ}$. To measure exactly the extent of the tumor embolus, a marked catheter should be used during portogram. To prevent intraperitoneal hemorrhage, it is crucial to embolize the liver parenchymal tract when the sheath is withdrawn.

The main limitation of the present study include the small number of patients, which limits the statistical analysis of the survival rate after operation between the patients with and without recurrence. Another limitation of this single-centre case series is its retrospective nature over a long time period. In addition, the cause of recurrence was not determined by pathological examination. Finally, this study does not objectively assess improvement in the quality of life of the patients.

In conclusion, interventional therapy has advantages of smaller injuries, well-tolerance, high success rate, quick palliation of symptoms and superior clinical efficacy in the treatment of venous vascular complications as a result of malignant tumors. However, whether interventional therapy increases the survival time of malignancy patients with venous vascular complications should be further investigated.

\section{Acknowledgements}

This study was supported by research grants from the Scientific Research Fund of Liaoning Science and Technology Agency, China (no. 2008225010-5) and the Scientific Research Fund of Liaoning Education Agency, China (no. 2007T183) and the Scientific Research Fund of First Hospital of China Medical University (no. FSFH1006).

\section{References}

1. Mewissen MW, Seabrook GR, Meissner MH, Cynamon J, Labropoulos $\mathrm{N}$ and Haughton SH: Catheter-directed thrombolysis for lower extremity deep venous thrombolysis: report of a national multicenter registry. Radiology 211: 39-49, 1999.

2. Kee ST, Kinoshita L, Razavi MK, Nyman UR, Semba CP and Dake MD: Superior vena cava syndrome: treatment with catheter-directed thrombolysis and endovascular stent placement. Radiology 206: 187-193, 1998.

3. AbuRahma A and Robinson PA: Effort subclavian vein thrombosis: evolution of management. J Endovasc Ther 7: 302-308, 2000.

4. Kreienberg PB, Chang BB, Darling RC III, et al: Long-term results in patients with thrombolysis, thoracic inlet decompression, and subclavian vein stenting for Paget-Schroetter syndrome. J Vasc Surg 33: S100-S105, 2001.

5. Kearon C, Kahn SR, Agnelli G, Goldhaber S, Raskob GE, Comerota AJ, American College of Chest Physicians: Antithrombotic therapy for venous thromboembolic disease: American College of Chest Physicians - evidence-based clinical practice guidelines, 8th edition. Chest 133: S454-S545, 2008.

6. Furui S, Sawada S, Kuramoto K, et al: Gianturco stent placement in malignant caval obstruction: analysis of factors for predicting the outcome. Radiology 195: 147-152, 1995.

7. Fletcher WS, Lakin PC, Prommier RF and Wilmarth T: Results of treatment of inferior vena cava syndrome with expandable metallic stents. Arch Surg 133: 935-938, 1998.

8. Lanciego C, Chacon JL, Julian A, et al: Stenting as first option for endovascular treatment of malignant superior vena cava syndrome. AJR Am J Roentgnenol 177: 585-593, 2001.

9. Lanciego C, Pangua C, Chacón JI, et al: Endovascular stenting as the first step in the overall management of malignant superior vena cava syndrome. AJR Am J Roentgnenol 193: 549-558, 2009.

10. Nagata T, Makutani S, Uchida H, et al: Follow-up results of 71 patients undergoing metallic stent placement for the treatment of a malignant obstruction of the superior vena cava. Cardiovasc Intervent Radiol 30: 959-967, 2007.

11. Kim HS, Preece SR, Black JH, Pham LD and Streiff MB: Safety of catheter-directed thrombolysis for deep venous thrombosis in cancer patients. J Vasc Surg 47: 388-394, 2008.

12. Maleux G, Marchal P, Palmers M, et al: Catheter-directed thrombolytic therapy for thoracic deep vein thrombosis is safe and effective in selected patients with and without cancer. Eur Radiol 20: 2293-2300, 2010.

13. Heymans S, Verhaeghe R, Stockx L and Collen D: Feasibility study of catheter-directed thrombolysis with recombinant staphylokinase in deep venous thrombosis. Thromb Haemost 79: 517-519, 1998.

14. Hutten BA, Prins MH, Gent M, Ginsberg J, Tijssen JG and Büller HR: Incidence of recurrent thromboembolic and bleeding complications among patients with venous thromboembolism in relation to both malignancy and achieved international normalized ratio: a retrospective analysis. J Clin Oncol 18: 3078-3083, 2000.

15. Prandoni P, Lensing AW, Piccioli A, et al: Recurrent venous thrombo-embolism and bleeding complications during anticoagulant treatment in patients with cancer and venous thrombosis. Blood 100: 3484-3488, 2002.

16. Nicholson AA, Ettles DF, Arnold A, Greenstone M and Dyet JF: Treatment of malignant superior vena cava obstruction: metal stents or radiation therapy. J Vasc Interv Radiol 8: 781-788, 1997.

17. Miller JH, McBride K, Little F and Price A: Malignant superior vena cava obstruction: stent placement via the subclavian route. Cardiovasc Intervent Radiol 23: 155-158, 2000.

18. de Gregorio Ariza MA, Gamboa P, Gimeno MJ, et al: Percutaneous treatment of superior vena cava syndrome using metallic stents. Eur Radiol 13: 853-862, 2003.

19. Stambo GW, Leto J, Van Epps K, Woeste T and George C: Endovascular treatment of intrahepatic inferior vena cava obstruction from malignant hepatocellular tumor thrombus utilizing Luminexx self-expanding nitinol stents. South Med J 99: 1148-1149, 2006.

20. Zamora CA, Sugimoto K, Mori T, et al: Use of the wallstent for symptomatic relief of malignant inferior vena cava obstructions. Radiat Med 23: 380-385, 2005. 
21. Kishi K, Sonomura T, Fujimoto H, et al: Physiologic effect of stent therapy for inferior vena cava obstruction due to malignant liver tumor. Cardiovasc Intervent Radiol 29: 75-83, 2006.

22. Nio Y, Iguchi C, Itakura M, et al: Placement of an expandable metallic stent improves the efficacy of chemoradiotherapy for pancreatic cancer with malignant portal vein stenosis or obstruction. Anticancer Res 29: 3329-3335, 2009.

23. Novellas S, Denys A, Bize P, et al: Palliative portal vein stent placement in malignant and symptomatic extrinsic portal vein stenosis or occlusion. Cardiovasc Intervent Radiol 32: 462-470, 2009.

24. Kishi K, Sonomura T, Mitsuzane K, et al: Self-expandable metallic stent therapy for superior vena cava syndrome: Clinical observations. Radiology 189: 531-535, 1993.
25. Smayra T, Otal P, Chabbert V, et al: Long-term results of endovascular stent placement in the superior cava venous system. Cardiovasc Intervent Radiol 24: 388-394, 2001.

26. Smith SL, Manhire AR and Clark DM: Delayed spontaneous superior vena cava perforation associated with a SVC Wallstent. Cardiovasc Intervent Radiol 24: 286-287, 2001.

27. Dinkel HP, Mettke B, Schmid F, Baumgartner I, Triller J and Do DD: Endovascular treatment of malignant superior vena cava syndrome: Is bilateral Wallstent placement superior to unilateral placement? J Endovasc Ther 10: 788-797, 2003. 